Swarthmore College

Works

Psychology Faculty Works

Psychology

2012

\title{
Therapeutic Communication From A Constructionist Standpoint
}

Kenneth J. Gergen

Swarthmore College, kgergen1@swarthmore.edu

M. M. Gergen

Follow this and additional works at: https://works.swarthmore.edu/fac-psychology

Part of the Psychology Commons

Let us know how access to these works benefits you

\section{Recommended Citation}

Kenneth J. Gergen and M. M. Gergen. (2012). "Therapeutic Communication From A Constructionist Standpoint". Discursive Perspectives In Therapeutic Practice. 65-82.

https://works.swarthmore.edu/fac-psychology/908

This work is brought to you for free by Swarthmore College Libraries' Works. It has been accepted for inclusion in Psychology Faculty Works by an authorized administrator of Works. For more information, please contact myworks@swarthmore.edu. 


\title{
Chapter 4
}

\section{Therapeutic communication from a constructionist standpoint}

\author{
Kenneth J. Gergen and Mary Gergen
}

Many therapists are familiar with the landscape of social constructionist thought. Indeed, many of those represented in the present volume have contributed significantly to its development. However, for those presently joining these explorations, some preliminary remarks may be helpful. Specifically, our attempt in this chapter is first to sketch out several major assumptions shared by those engaged in constructionist endeavours. After describing some of these central ideas, we will take up the challenge of therapeutic communication. Here we will move beyond existing accounts to demonstrate what might be called a radical relationalism that constructionism invites. Finally, we shall explore significant implications of this account for therapeutic practice.

\section{The social construction of the real and the good}

There are many ways to tell the story of social constructionism. Each account would construct constructionism in a different way; each would be useful in a different context. In what follows we shall briefly describe three of the most widely shared narratives circulating today. These accounts are elaborated more fully elsewhere. ${ }^{1}$ We have selected these particular stories because they are simultaneously among the most unsettling and profoundly liberating. They also relate most clearly to the discursive theme that links together the chapters of the present book.

\section{The social lodgment of knowledge}

Perhaps the most generative idea emerging from the constructionist dialogues is that what we take to be knowledge finds its origins in human relationships. What we believe to be true as opposed to false, objective as opposed to subjective, scientific as opposed to mythological, rational as opposed to irrational, moral as opposed to immoral is brought into being by historically and culturally situated groups of people. This view stands in dramatic contrast to two of the most important intellectual and cultural traditions of the West: the individualist and the communal. On the one hand is the tradition of the individual knower - the rational, self-directing, morally centred,

1 See, for example, Gergen (2009a), and Gergen and Gergen (2004). 
and knowledgeable agent of action. In effect, the constructionist dialogues challenge the longstanding individualist tradition and invite an appreciation of relationship as central to human well-being. It is not the individual mind in which knowledge, reason, emotion, and morality reside, but in relationships. ${ }^{2}$

The second view of knowledge, that is the communal view, is also challenged. The possibility that the accounts of scientists, or any other group, reveal or approach the objective truth about what is the case is cast into doubt. In effect, propose the constructionists, no one arrangement of words, mathematical formulae, or symbolic system is necessarily more objective or accurate in its depiction of reality than any other. To be sure, accuracy may be achieved within a given community or traditionaccording to its rules and practices. Physics and chemistry generate useful truths from within their communal traditions, just as psychologists, sociologists, novelists, and priests do from within theirs. But from these often competing traditions we cannot locate a transcendent truth, a 'truly true'. Any attempt to determine the superior account would itself be the outcome of a given community of agreement. All claims to Truth must be viewed from some perspective, and beyond any perspective, one has no resources for speaking at all.

Unsurprisingly, these arguments have provoked strong and sometimes angry reactions among scientific communities in particular. Let's say you have devoted a lifetime to pursuing what you believe to be objective knowledge, and you despair of the unverifiable myths, credos, and folk beliefs by which common people lead their lives. Under these conditions it is difficult to be told that science is itself a social construction and not intrinsically superior to other traditions. Yet, it is our view that such anguish is based on a misreading of the constructionist message. Western medical science, for example, does indeed offer useful truths; most of us would scarcely wish to abandon them. However, these truths are based on an enormous array of culturally and historically specific constructions, for example, about what constitutes an impairment, health and illness, life and death, the boundaries of the body, the nature of pain, and so on. When these assumptions are treated as universal-true for all cultures and times - alternative conceptions are undermined and destroyed. To understand death, for example, as merely the termination of biological functioning would be an enormous impoverishment of human existence. The point is not to abandon medical science, but to understand it as a cultural tradition-one among many.

Thus, social constructionism first serves an enormous liberating function. It removes the rhetorical power of anyone or any group claiming truth, wisdom, or ethics of universal scope-necessary for all. In this sense, social constructionist ideas tend to support those who would speak out against the dominant discourse. All voices may justifiably contribute to the dialogues on which our futures depend. It is also important to understand that although knowledge claims are socially constructed, this does not render them insignificant. Again, it is to recognize that each tradition, while limited, may offer us options for living together. In this way constructionism invites a posture of infinite curiosity, where every tradition may offer us riches, and new amalgams

2 For a more extended account of both the critique of individualism, and its relational replacement, see Gergen (2009b). 
stand ever open to development. At the same time, because all traditions will tend so suppress, all should be open to critical reflection. Every voice has the potential for both good and evil from some perspective. The future of the planet depends importantly on how disparate or conflicting discourses may be reconciled, amalgamated, or transformed.

\section{The centrality of discourse}

Many scholars believe that Ludwig Wittgenstein was the most significant philosopher of the 20th century. After reading his later work, most especially Philosophical Investigations (1953), one can never see the aim of philosophy in the same way again. In large measure this is because Wittgenstein's work challenged the capacity of philosophy to yield true understanding of knowledge, rationality, ethics, the self, and all the other subjects of longstanding concern to philosophers. As Wittgenstein proposed, our descriptions and explanations of the world are formed within language, or what he calls 'language games'. Games of language are essentially conducted in a rule-like fashion; to make sense at all requires that one play by the rules. The rules of grammar present the most obvious case; but there are also myriad other rules. For example, it is not acceptable for me to say that 'my love is oblong'. The utterance is grammatically correct, and it cannot be falsified with empirical data. Rather, our ways of talking about love in the 21 st century do not happen to include the adjective 'oblong'. Expanding on this point, we can describe the major questions asked by philosophers as language games. For example, the longstanding question of whether the mind truly has access to the external world - the 'problem of epistemology' as it is called-is a problem only within a given game of language. To play the game we must agree that there is a 'mental world' on the one hand and a 'material world' on the other (an 'in here' and an 'out there'), and that the former may possibly reflect the latter. If you do not agree to play by these rules, there is no 'problem of individual knowledge'. It is dissolved.

Yet, to view language as simply a game is limited. As Wittgenstein proposed, language use is lodged within broader 'forms of life', as he called them. Consider the form of life we call a 'soccer match'. To be sure, there are traditional ways of talking about soccer-about teams, scores, penalty kicks, and so on. But these forms of talk are embedded within forms of action and material. One cannot simply yell out 'Score!' on a busy street corner, without arousing suspicion. There are only specific conditions in which such a cry makes sense, and this depends on a specific array of objects (such as the playing field and the ball) and people (such as the players and referees).

Wittgenstein's ideas are highly congenial with the view of knowledge as social in origin. As people coordinate their actions, a major outcome is often a system of signals or words. The words often serve to name the world for the participants. This is 'a goal', you have the 'flu', that is 'a stolen car', and so on. These words are enormously important to sustaining our daily relationships. Not only do they represent the agreements regarding what exists for the participants, but they essentially constitute the glue by which their very forms of life-or traditions - are held together. What sense is there in a jury trial without a language of guilt and innocence; what would the profession of psychology be without a language of the mind; and what would become of religion if we abandoned the language of the spirit? 


\section{Objectivity as ideology}

Social constructionism shares much with a pragmatic view of knowledge claims. That is, traditional issues of truth and objectivity are replaced with concerns with practical outcomes. It is not whether an account is true from a god's eye view that matters; rather we ask what will happen if we take any truth claim seriously. There can be many truths, depending on community traditions, but as the constructionist asks, what happens to us - for good or ill -if we accept one as opposed to another account? There are no meaningful words without consequence. In this sense, the increased awareness of the communal construction of the real and the good does far more than unsettle our traditional beliefs in truth, objectivity, and knowledge-beyond history and culture. Placed in question is also the right of any particular group-scientific or otherwise-to claim ultimate authority of knowledge. And with such questioning, we are also invited into deliberation on what we judge to be worthwhile, good, or desirable

Such a conclusion has had enormous repercussions in the academic community and beyond. This is so especially for scholars and practitioners concerned with social injustice, oppression, and the marginalization of minority groups in society. If communities create realities (facts and good reasons) congenial to their own traditions, and these realities are established as true and good for all, then alternative traditions may be obliterated. Regardless of whether we are speaking of scientific fact, canons of logic, foundations of law, or spiritual truths, as we formulate the world we implicitly favour certain ways of life over others. Thus, for example, the scientist may use the most rigorous methods of testing intelligence, and amass tomes of data that indicate racial differences in intelligence. However, to presume that there is something called 'human intelligence,' that people differ in their possession of this capacity, that there is something called 'race', that race can be clearly determined, and that a series of question and answer games can reveal one's intelligence or race, is all specific to a given tradition or paradigm. Such concepts and measures are not required by 'the way the world is'. Most importantly, merely entering the paradigm and moving within the tradition is deeply injurious to those people classified as inferior by its standards. Or to put it another way, the longstanding distinction between facts and valuesobjective reflections of the world, and subjective desires or feelings of 'ought'-cannot be sustained. Rather, values are intrinsic to facts.

As we see, sensitivity to the politics of the real and the good invites a broad critical posture. The interested reader may wish to explore such critiques and their rejoinders. ${ }^{3}$ Unfortunately, however, many of those drawn into a critical posture simply remain there. The gadfly rarely becomes a butterfly. To understand the politics of knowledge also opens the door to appreciation. It is not simply 'what we lose' within any tradition of knowledge that is important, but what we gain as well. We may ask of all claims to knowledge, wisdom, insight, and the like, 'what follows', 'who benefits', and 'who is silenced?'. All constructions will place limits upon our lives; but without construction there is nothing worthy of any pursuit. From these new amalgams we may move towards richer and more inclusive forms of life.

3 See for example, Parker (1998) and Nightingale and Cromby (1999). 
These three themes - centring on the social construction of the real and the good, the pivotal function of language in creating intelligible worlds, and the political and pragmatic nature of discourse-have rippled across the academic disciplines and throughout many domains of human practice. To be sure, all such developments are controversial. However, such ideas also possess enormous potential. Such is the case in therapy as it is in the global context. The therapeutic community has long participated in the constructionist dialogues and richly extended their potential. The present volume, for example, brings into common dialogue the voices of narrative, collaborative, and brief therapists - among others-and suggests a broad sea-change in thought and practice. However, the full implications of a constructionist orientation remain unclear. It is within this context, that we now explore the topic of therapeutic communication. The process is of singular significance, not only to the outcomes of therapy, but to the very idea of a constructed world. This discussion will also help to clarify differences between constructionism and contrasting traditions, and to appreciate its radically relational character. After treating some of the rudiments of the communication process we turn to therapeutic implications.

\section{Therapeutic communication in question}

The question of what it is in the therapeutic encounter that brings about change has been central to the endeavour since its very inception. Perhaps the central candidate for answering this question is therapeutic communication. There is something about the nature of communicative interchange that seems pivotal to the change process. Yet, such an answer is scarcely sufficient. How are we to understand the process of communication? What precisely is it about communication that brings about transformation? What forms of communication are invited; how might we be more effective? There are significant differences among schools of therapy in their approach to communication, and they are highly consequential. Consider the client who complains of his lack of sexual desire. If one is a marital counsellor, these words may be treated as an accurate representation of reality, and the basis of a programme of support. In contrast, if one is a psychoanalyst, the content of the client's account might be disregarded, and his words examined for messages from the domain of the unconscious. For the cognitive-behavioural therapist, however, the same words are neither descriptions of the real world nor manifestations of repressed desires, but indicators of the world from the client's perspective. The therapist might thus launch inquiry into the logic of this perspective, and its possible distortions. And, for the structuralist family therapist, the client's words may be understood in none of these ways, but as indications of the configuration of family relations. In this case the therapist might address the ways this expressed lack of desire is related to the actions of other family members. Each presumption about the nature of language and the process of communication yields a different therapeutic posture.

In what follows we first consider several major assumptions that underlie most therapeutic practices developed to date. Although there is much to be said on behalf of these assumptions, in each case we shall single out major shortcomings. While our conceptual heritage is rich, traditional assumptions about therapeutic communication 
erect barriers beyond which our practices cannot proceed. We shall then sketch out the rudiments of a constructionist theory of communication. In this account we find a dramatic disjunction with the past and a shift to a radical relationalism. We then present significant implications for therapeutic practice. Consider, then, several traditional assumptions and the critical problems they create:

\section{The realist assumption}

One of the most broadly shared views of language is based on the assumption that words are (or can be) reflectors of the real. That is, language can (and should) function so as to provide accurate accounts of what is the case. This is the view inherited by most of the sciences, as they set out to replace misleading, fallacious, or superstitious beliefs with true and accurate accounts of the world. For many therapists it is also essential to distinguish between client accounts that are accurate, realistic, and truthful, versus those that are distorted, fanciful, or duplicitous. The realist assumption is also central to those attempting to develop diagnostic categories and measures of pathology. In daily life it is a view that lends support to the distinction between objective facts and subjective opinions, and moral weight to demands that people 'tell the truth'.

There is much to be said about the importance of this tradition both to scientific and cultural life. However, as the preceding discussion makes clear, the realist assumption is deeply flawed. There is no privileged relationship between a given language and the state of things; there is no particular arrangement of words and phrases that is uniquely tailored to the 'world as it is'. Rather, as we have seen, declarations of the real and the true are always located within relationships-friendships, families, communities, and traditions. Within these relationships there can be undisputed realities- 'myocardial infarction' in medicine, a 'three-point shot' in basketball, and so on. In this sense, to tell a lie is not to misrepresent the world, but to violate a communal tradition.

\section{The subjectivist assumption}

Often coupled with the realist assumption is a second view of longstanding. As it is typically said, we each exist in our own private worlds of experience, a mind set apart from, and reflecting upon nature-a state of subjectivity that variously grapples with understanding the conditions of the objective world. On this account, the words we speak are held to be outer expressions of the inner world, the subjective mind made manifest. This view has played a major role in science, as we count the scientist's words as representing his or her experience of the world, and demand that observations be shared to insure agreement among subjectivities ('objectivity' as shared 'subjectivity'). The assumption is critical to most schools of therapy in the past century. In almost all cases we listen to a client's language as an outer expression of private experience (or, as in the Freudian case, that which lies beneath conscious experience to give it shape). And, the assumption is a common feature in daily relations, as we speak of the difficulties in knowing what others mean by their words, or how they 'really feel'. Intimacy, we believe, is a reflection of the closeness of two otherwise independent subjectivities. 
Here we touch briefly on only two problems of subjectivity, the first conceptual and the second ideological. On the conceptual level, it is important to realize that no one has yet been able to give a defensible account of how a person's words give us access to his or her inner world. Given another's utterances, we have no way of knowing what they say about the speaker's subjective state. Hermeneutic theorists, concerned with how it is we can accurately understand the intentions behind the words of the Bible or holy writs, have worried about the problem of 'inner access' for over three centuries now. A satisfactory answer to this question has never been forthcoming. In Hans Georg Gadamer's (1975) pivotal work, Truth and Method, the major emphasis shifts to the 'horizon of understanding' that the reader inevitably brings to the text. As Gadamer reasoned, all readings must necessarily draw from this forestructure of understanding - what it is the reader presumes about the world, the writing, the author, and so on. And reading must inevitably take place from this horizon. Much the same conclusion is reached by a host of 'reader response' theorists in literary studies. As Stanley Fish (1980) has put the case, every reader is a member of some interpretive community, a network of people who understand the world in certain ways. And whatever interpretation of the text is made will inevitably rely on these understandings. In effect, the reader never makes authentic connection with the subjectivity of the writer; there is no escape from the standpoint one brings to the interpretation.

The dismal conclusion of this line of criticism is that we never gain access to the other's subjectivity; we never understand each other! We shall revisit this problem shortly. However, there is a second line of attack on the subjectivity assumption, one that resonates with our earlier discussion of the politics of knowledge. Here it is variously proposed that by placing such importance on individual subjectivity we give further support to an individualist ideology, an ideology detrimental to our cultural future. To reiterate some of the earlier critiques, when we hold individual subjectivity as the essential ingredient of the person, we simultaneously construct a world of fundamentally isolated individuals, each locked within their own private world. All we have to count on, ultimately, is ourselves. Others are by nature alien and because self-seeking is the obvious choice under such conditions, others may indeed be seen as potential enemies. When the quality of individual subjectivity is paramount, all forms of relationship - marriage, friendship, family, and community - are necessarily artificial and secondary. If this form of ideology retains its pervasive grip on cultural life, the future seems grim. In effect, the subjectivist assumption is socially corrosive.

\section{The strategic assumption}

There is a third problematic assumption regarding communication, one often made by therapists in particular. It is frequently held that communication operates as the major means by which individuals influence each other's actions. More specifically, it is reasoned, each of us uses language to achieve our goals, satisfy our desires, etc. Because of the complexities of daily life, we must rationally consider what we can say, when, where and to whom. Language typically functions, then, as a strategic implement through which we achieve their goals. It is in this sense, as well, that the therapist 
may select his/her words carefully, insert them into the conversation at the proper juncture in order to change to client or the pattern of family relations.

In light of the preceding discussion, the problems of the strategic assumption require but brief attention. For one, the position borrows heavily from the subjectivist tradition-I I desire and plan, and therefore I speak'. In this sense, the strategic assumption suffers from the same conceptual enigmas and the ideological shortcomings just discussed. Private goals are pre-eminent; others become secondary, mere utilities in the service of self. When we play out the implications of the strategic assumption the critique is intensified. When we understand communication as primarily serving private ends, human relations become a sea of manipulation. When we view communication in this way, acts of trust seem naïve, commitment a sign of weakness, and the pursuit of human rights little more than a political ploy. Even the therapist undermines his/ her credibility, as the motive behind his or her communication to the client becomes suspect The therapist may be viewed as a master manipulator, and clients may come to see themselves as mere pawns. A strategic orientation can be fractionating.

\section{Communication as collaborative action}

The question we now address is whether an alternative conception of human communication can be developed. And, can such a conception avoid repeating the problems inherent in the earlier traditions? In our view, an alternative view of human communication can indeed be drawn from the constructionist dialogues, not only as they are taking place within therapeutic circles, but as they have developed in the neighbouring domains of ethnomethodology, the history of science, the sociology of knowledge, and literary theory. ${ }^{4}$ In each of these cases there is a strong tendency to place the locus of meaning within the process of interaction itself. That is, the individual agent is de-emphasized as the source of meaning; attention moves from the within to the between. Yet, how are we to understand such a move, and what are the action implications? For purposes of furthering the dialogue, in what follows we make a preliminary incursion into these domains. We offer here a series of rudimentary propositions that place meaning squarely within the relational matrix:

\section{Individual utterances possess no meaning}

We pass each other on the street. I smile and say, 'Hello, Anna'. You walk past without hearing. Under such conditions, what have I said? To be sure, I have uttered two words. However for all the difference it makes I might have chosen two nonsense syllables. You pass and I say 'Umtot gigen...' You hear nothing. When you fail to acknowledge me in any way, all words become equivalent. In an important sense, nothing has been said at all. I cannot possess meaning alone. ${ }^{5}$

4 See, for example, Garfinkel (1967); Kuhn (1970); Latour (1987); Edwards and Potter (1994); Fish (1980); and Shotter, (1993).

5 One may object: 'well, even if not acknowledged, what I say might mean something to me personally', and that may be. But the question then becomes, how did your utterances come to have personal meaning? We take up this issue shortly. 


\section{Meaning is realized through supplementary action}

Lone utterances begin to acquire meaning when another (or others) coordinate themselves to the utterance, that is, when they add some form of supplementary action (whether linguistic or otherwise). Effectively, I have greeted Anna only by virtue of her response. 'Oh, hi, good morning...' brings me to life as one who has greeted. Supplements may be very simple, as simple as a nod of affirmation that indeed you have said something meaningful. It may take the form of an action, e.g. shifting the line of gaze upon hearing the word, 'look!'. Or it may extend the utterance in some way, as in 'Yes, but I also think that...'. We thus find that to communicate at all is to be granted by others a privilege of meaning. If others do not treat one's utterances as communication, if they fail to coordinate themselves around the offering, one is reduced to nonsense.

To combine these first two proposals, we see that meaning does not reside within either individual, but within the relationship. Both act and supplement must be coordinated in order for meaning to occur. Like a handshake, a kiss, or a tango, the individual's actions alone are insufficient. Communication is inherently collaborative. In this way we see that none of the words that comprise our vocabulary have meaning in themselves. They are granted the capacity to mean by virtue of the way they are coordinated with other words and actions. Indeed, our entire vocabulary of the individual-who thinks, feels, wants, hopes, and so on-is granted meaning only by virtue of coordinated activities among people. The birth of 'myself lies within relationship.

\section{Supplementary action is itself a candidate for meaning}

Any supplement functions twice, first in granting significance to what has preceded and second as an action that also requires supplementation. In effect, the meaning it grants remains suspended until it too is supplemented. Consider a client who speaks of her deep depression; she finds herself unable to cope with an aggressive husband and an intolerable job situation. The therapist can grant this report meaning as an expression of depression, by responding, 'Yes, I can see why you might feel this way; tell me more about your relationship with your husband'. However, this supplement, too, stands idle of meaning until the client provides the supplement. If the client ignored the statement, for example, going on to talk about her success as a mother, the therapist's words would be denied significance. More broadly, we may say that in daily life there are no acts in themselves, that is, actions that are not simultaneously supplements to what has preceded. Whatever we do or say takes place within a temporal context that gives meaning to what has preceded, while simultaneously forming an invitation to further supplementation.

\section{Acts create the possibility for meaning but simultaneously constrain its potential}

If one gives a lecture on narrative therapy, this lecture is meaningless without an audience that listens, deliberates, affirms, or questions what one has said. In this sense, every speaker owes to his or her audience a debt of gratitude; without their engagement the speaker ceases to exist. At the same time, the lecture creates the very possibility for 
the audience to grant meaning. While the audience creates the speaker as a meaningful agent, the speaker simultaneously grants to them the capacity to create. They are without existence until there is an action that invites them into being.

Yet, it is also important to realize that in practice, actions also set constraints upon supplementation. If one speaks on narrative therapy, audience members cannot supplement in any way they wish. They may ask a question about object relations theory, but not astrophysics; comment on the concept of externalization but not on the taste of radishes. Such constraints exist because the lecture is already embedded within a tradition of act and supplement. It has been granted meaning as a lecture on narrative therapy,' by virtue of previous generations of meaning makers. In this sense, actions embedded within relationships have prefigurative potential. The history of usage enables them to invite or suggest certain supplements as opposed to others-because only these supplements are considered sensible or meaningful within the tradition. Thus, as we speak with each other, we also begin to set limits on each other's being; to remain in the conversation is not only to respect a tradition, but to accede to being one kind of person as opposed to another.

\section{Traditions of coordination furnish the major potentials for meaning}

To amplify a preceding line of reasoning, it is important to recognize that the words and actions upon which we rely to generate meaning together are largely byproducts of the past. If another approached you and began to utter a string of vowels, 'ahhh, ehhhh, ooooo, uuuu...' you would surely be puzzled; perhaps you would make for an exit, as this individual might well be dangerous. This is so because this utterance is nonsense, or to put it another way, not recognizable as a candidate for meaning within Western traditions of coordination. Similarly, if you began to dance with someone, and he or she suddenly crouched and gazed at the floor, you would scarcely continue dancing. Such actions are not part of any coordinated sequences with which you are familiar. Our capacity to make meaning together today thus relies on a history, often a history of centuries' duration. We owe to traditions of coordination our capacities for being in love, demonstrating for a just cause, or taking pleasure in our children's development.

Most often, our traditions of coordination are not recognized as traditions by the actors. They simply become a 'natural' way of life. This is so even with patterns that are detrimental to most relationships, such as relational patterns involving anger, blame, criticism, disinterest, jealousy, contempt, and so on. This is scarcely to say that there is no room for novel words and actions. Indeed, in the past century we have witnessed an explosion in new vocabulary terms, sporting activities, dance steps, and so on. Because we are not determined by the past, we are free to play, to violate expectations, and to explore the outrageous. This is indeed a major challenge for the therapeutic profession: collaborating in the generation of new and more viable forms of action.

\section{Meanings are subject to continuous reinterpretation}

In light of the above, we find that what an utterance means is inherently undecidable. No amount of discussion, discourse analysis, conversation analysis, or other attempt 
to determine what has been said, can be conclusive. The meaning of any utterance is a temporary achievement, born of the collaborative moment. Further, as relations continue over time, what is meant stands subject to continuous alteration through an expanding arena of action/supplements. Sarah and Robert may find themselves frequently laughing together-affirming each other as humorous persons - until Robert announces that Sarah's laughter is 'unnatural and forced', just her attempt to present herself as an 'easy going person' (in which case the definition of the previous actions would be altered). Or Sarah could announce, 'This is all very pleasant, Robert, but really you are so superficial; we really don't communicate at all' (thus reducing Robert's humour to banality). At the same time, these latter moves within the ongoing sequence are subject to further reconstitution. (In reply to Robert's accusation of being unnatural, Sarah replies, 'Robert, are you worried about your job again? What's bothering you?'. Or, Robert replies to Sarah's ascription superficiality with, 'Now I see... You are only saying that, Sarah, because you find Bill so attractive'.) Such instances of alteration may also be far removed from the interchange itself (e.g. consider a divorcing pair who retrospectively redefine their entire marital trajectory), and are subject to continuous change through interaction with and among others (e.g. friends, relatives, therapists, the media, etc.).

In summary, we find the exclusive focus on the face-to-face relationship is far too narrow. For whether one 'makes sense' is not under one's control; nor is it determined by others, or fixed by the collaborative process in which meaning struggles towards realization. At the outset, we largely derive our potential for coordination from our previous immersion in a range of other relationships. We arrive in the relationship as extensions of the past. And, as the current relationship unfolds, it serves to reform the meaning of the past. These interchanges may be supplemented and transformed by still others in the future. In effect, meaningful communication in any given relationship ultimately depends on an extended array of relationships, not only 'right here, right now,' but how it is that you and I are related to a variety of other persons, and they to still others-and ultimately, one may say, to the relational conditions of society as a whole. We are all in this way interdependently interlinked - without the capacity to mean anything, to possess an 'I'- except for the existence of an extended world of relationship.

\section{Therapy as collaborative action}

Developed here is a particular way of understanding the process of communication from which meaning emerges, is sustained and transformed. This account avoids certain pitfalls of most traditional accounts, and simultaneously realizes some of the potentials of constructionist reasoning. It is important to point out that we are not saying that we have explicated 'the true nature of communication'. Rather, this view is offered in the spirit of constructionism itself; it is simply an alternative way of making communication intelligible. The question is not whether it reveals the 'reality of communication,' but what follows from such an exposition? In what way would such an understanding alter any of our practices, and would such alterations be useful for the therapeutic venture? To be sure, such questions cannot be answered all at once. 
There are many implications, both great and small, and further dialogue is needed to glimpse the potentials and problems. Some of the implications of this collaborative account are already well integrated into practice; other implications may prove too radical for contemporary application. However, to open discussion on therapeutic communication as collaborative action, we offer the following points:

\section{There is no mental illness in itself}

Because no human expression comes into meaning save through others' supplements, there is no suffering and no mental illness prior to collaboration. To be sure, one may 'feel depressed,' or encounter an 'obvious schizophrenic'. But the fact that one 'feels depressed' is already prepared by a previous immersion in a culture of circulating meaning. (Prior to the 20th century I could not 'feel depressed,' because the very intelligibility of depression only emerged within this century.) In the same way we 'see schizophrenics,' because there are already participants in a culture that collaborates to create the meaning of 'mental disease'. Here it is important to lay stress on the responsibilities of the therapist in creating and/or sustaining the life of anguish and illness within the therapeutic relationship. The therapist functions as a major collaborator in the generation of meaning; whether the client is anguished or ill, resourceful or resilient, is importantly dependent on the continuing collaborative process. It is in this light that deliberation is imperative on the movement towards increasing diagnosis, neurological explanation, and pharmacological 'cures'. Such constructions represent both an extension of a singular, medical model of human problems, and a Western ideology of individualism. Together they function to limit - in many unfortunate ways-conversations about human maladjustments and misery. ${ }^{6}$

\section{There is no therapeutic treatment in itself}

Although the therapist bears some responsibility for how clients come to understand themselves, their feelings, their relationships and so on, they are not omnipotent. Their supplements to clients' statements do not acquire meaning until supplemented by the client in turn. To put it more broadly, there is no 'therapeutic treatment' in itself; the actions that we might normally describe as 'therapeutic' do not become so until clients supplement the actions of the therapist in this regard. Custodians in mental hospitals know this very well. Often their honest attempts to help patients produce angry and resentful responses. Good treatment from the custodian's perspective may be regarded as manipulation and control by the patient. We must ask, then, whether we should reconsider what we, as professionals, call 'good treatment'. If the concept of 'good treatment' is not collaboratively generated, then 'therapeutic help' becomes unlikely.

\section{Understanding a client is a form of collaborative action}

We inherit a psychological view of interpersonal understanding. As we say, understanding occurs when subjectivities are linked or reflect each other accurately.

6 For further discussion of these issues, see Gergen (2006). 
Earlier we located important flaws in this view. As argued, if understanding were a matter of intersubjective synchrony, we would never understand each other. Yet, we do believe that understanding occurs, and we are certain when another misunderstands us. How can we account for understanding from the collaborative view? Here it is useful to view understanding not as a mental activity, but as a particular form of supplementation. To be 'understanding' is to coordinate one's actions to those of another; it is to be a certain kind of person in relation to the other. If another speaks of their anguish, a listener 'understands' when he or she responds in a particular way, with certain words, tones of voice, and gestures as opposed to others. If the listener simply gazed out the window during the tale of sadness, the speaker might justifiably say, 'You just don't understand, do you?'. There is no peering into the mind of the other here; there is only coordinated action within a tradition.

\section{The effective therapist is a skilled coordinator}

What is actually changed through therapy? Consistent with its individualist base, this question is typically answered in terms of the individual psyche. It is through the removal of repression, a process of catharsis, a gain in insight, the enhancement of self-esteem, or the alteration in cognitive schemas, as it is variously reasoned, that long-term change is effected. From the present perspective the landscape is dramatically altered. The psychological condition is not the centre of concern; it is relational existence. The individual arrives in therapy as a participant in a relational network, a network that extends outward from intimates to the culture at large, and backward in time to pre-existing relationships and traditions. It is this matrix of relationship out of which 'the problem' is created and designated as a problem. The therapeutic relationship represents the establishment of a new coordination, a coordination that will develop from the resources that both the therapist and client bring to the relationship. The major challenge confronting the therapeutic relationship is whether the collaborative trajectory of client/therapist can unsettle or transform the generative matrix in such a way that the problem is resolved, dissolved, or reconstructed.

In this sense we see that the therapist's most valuable resources are conversational actions. In the same way that skilled basketball players possess a rich vocabulary of actions enabling them to score, so are skilled therapists those who are able to coordinate effectively with the client in such a way that agreeable outcomes may be achieved within the extended matrix. It is not the storehouse of facts, concepts, distinctions and so on that the therapist has at his or her disposal that counts, but the capacity for flexibility in relationship. 'Knowing how' as opposed to 'knowing that'. This relational capacity will surely be verbal. Required are capacities to move in narrative, metaphor, exploration, irony, humour, pathos, curiosity, imagination, and much more. Yet, it is not simply the language content that is important. Posture, gaze, tone of voice, facial expression, gait, and so on all contribute to the form and ramifications of relationship. All may be used to unsettle or transform the matrix. All may provide the client with models for action outside the therapeutic relationship.

Because therapy is inherently a coordination, and no two clients will enter from the same relational matrix, there should be no hard and fast rules for the therapeutic encounter. Specific techniques or canons of therapeutic practice will only narrow the 
capacities for coordination. If the client recognizes the therapist's utterances as 'technique', they may indeed be disregarded or resented. If the therapist must ask questions according to some manual of prescribed practice, only dis-ease will follow. Thus, there is no unequivocal answer to a therapist's question of 'how should I proceed?'. The same words and phrases, useful in one context, may be crippling in another. Again the basketball analogy is useful; with experience the skilled player develops a repertoire of usable actions. There are no rules for which action is most effective; the conditions of play are complex and rapidly changing. The skilled player is one who can rapidly draw from the repertoire as the 'conversation of the court' unfolds. A skilled player can 'unsettle' the opponent's 'traditions of action'. At the same time the opponents will also model these skills and become more proficient. The patterns of play continue to unfold. The therapeutic encounter shares the same potential for innovative activity. In the case of therapy, however, there are no opponents; there is only the collective construction of well-being at stake.

\section{A major challenge is to enter conversations already solidified}

When working with a concept of meaning as relational coordination there is a strong tendency to stress the here and now-' 'us talking together at this moment'. It is in the present moment that meaning is under construction, and thus the future in the balance. Yet, this focus often obscures the ways in which the resources imported into the therapeutic session are anchored in relational history. This history can stand as a major impediment to change. On the simplest level, people develop modes of talking and acting that are comfortable and reliable. In an important sense, they are skilled in these forms of action. Thus, depressive, angry, or self-critical styles of being may seem dysfunctional from the therapist's standpoint. However just such modes of action may serve as reliable, 'natural,' and finely honed skills for the client. 'I know so well how to attack others for their shortcomings,' a client may say. 'Even if others avoid me for this reason, this is what I know how to do'. Without new skills or performance capacities in hand, a client may scarcely relinquish the old.

On a more subtle level, all of us carry with us residues of past relational patterns. As proposed earlier, it is the private recirculation of these residues that we speak of as 'thought' or 'emotion'. Such forms of recirculation may establish themselves as recurring scenarios. There is the voice, for example, that says, 'You are no good', and a following one that gives you a lift, 'Yes you are, you are great!'. You perform with the latter voice at hand and things go well. The cycle repeats, and you develop what might be called a private'coping scenario'. Such privately re-circulated scenarios may not always be so functional, as in 'You are no good... I will hurt you for saying that. . . but everybody agrees that you're no good... I will fight them all...'. Performances derived from such a scenario may be suspicious and hostile. These privately re-circulated and well-worn scenarios may be the most difficult to interrupt. Anyone who has worked with what we commonly label as 'eating disorders' has encountered the strangulating grip of the private conversation. In our view, one of the most challenging problems confronting contemporary therapy is that of linking the face-to-face conversation with the client to the off-stage scenarios. 


\section{Meaning is subject to continuous transformation}

From the collaborative perspective, each move within a conversation grants meaning to what has preceded. The meaning of all our words and actions is importantly dependent on those who respond to them. And their responses are denied significance until they too are supplemented. In effect, meaning is always in process, never complete, forever open to the next move in the conversation. This is to say that any attempt to specify the meaning of a past action - 'what I intended. ..', 'what I was trying to say...', 'what you did to me...', 'what this meant to me...'-is itself a supplement that transforms the past. Such attempts give the past shape and consequence that could not be acquired save through the attempt itself. And these attempts as well stand mute until supplemented. There is no final moment of illumination, a moment at which meaning becomes indelible and undeniable.

The therapeutic implications of this reasoning are many. First, and to underscore an earlier argument, any therapeutic interpretation of a client's words or actions create the meaning of those words and actions. Any attempts by the therapist to point out continuities or discontinuities within the client's life, are themselves creations of continuity and discontinuity. Further, any attempt by the client to speak about the past, to reveal its secrets, to render it meaningful is itself a transformation of the past. Amplifying Donald Spence's (1982) arguments, this is to make prominent the significance of narrative as opposed to historical truth. Accounts of the past are created within the conversational space of the present.

Returning to an earlier issue, it also follows that all attempts at psychodiagnostic testing and therapeutic outcome assessment are essentially transformations of meaning. In both cases, whatever has occurred, whatever has been said or done, is granted a certain meaning that it did not itself possess. There is no pathology until the testing instrument has transformed the individual's words and actions into pathology. There is no positive or negative outcome of therapy until the assessment device renders certain patterns meaningful as outcomes. Important questions must thus be addressed: for whom is this pathology, what makes this diagnostic category more useful than another; are illness categories useful for clients; who is deciding on what constitutes a 'good outcome', what clients and therapists are benefited (or marginalized) by a given conception of outcome; whose voices are allowed into the conversation; and when does the conversation terminate? Again, from the view of communication as coordination, the practices of diagnosis and outcome assessment should be opened to full re-examination.

\section{A major challenge is bridging the gap between meaning-making within the therapeutic relationship and the worlds outside}

If we follow the collaborative logic, therapy represents a conversation in which participants borrow heavily from their relations outside, but in which they simultaneously create the grounds for a new and unique reality (discourse patterns shared by them alone.) Under these conditions it might be possible for therapist and client to locate a wonderfully agreeable mode of relating - a shared sense of harmony and fulfilment 
within the encounter. However, this same reality may also be wholly contained within the relationship. That is, it may have little or no 'street value', little transportability into other relations. Given the collaborative view, the major question is whether the conversational resources generated within the therapeutic relationship can be transported outside of this context. Can the metaphors, narratives, deconstructions, re-framings, multiple selves, expressive skills, and so on developed within the therapeutic encounter be carried into other relations in such a way that these relations are usefully transformed?

At one level, it is easy enough to conclude that such reverberations do occur. However, more effective demonstrations of the ways in which therapeutic conversations actually insinuate themselves into the life worlds of clients would be very useful. Further, more concerted attention is needed to how the contexts of therapy and other life worlds can be made to converge. The most obvious means, and one very congenial to the family therapy movement, is to work with relationships rather than single individuals. In this way new discursive forms and practices are set directly in motion. However, this does not fully solve the problem, as the group reality of the therapeutic hour may not be transportable; family members are also embedded in multiple relationships outside the family circle. The family in the therapy room is not the same family at the dinner table.

\section{In order to remain effective, therapeutic practices must be continuously transformed}

For over a century now, therapists have searched for 'the cure' to the problems confronted by their clients. It is thus that we have witnessed a parade of therapeutic schools, each eager to champion their particular form of treatment, and typically dismissing the remaining field of contenders. In the USA, there are longstanding attempts to assess the comparative efficacy of various practices, thus to winnow out the 'mere pretenders'. In other nations, the laws only recognize a narrow range of schools as worthy of health insurance coverage; the remaining are left to perish.

When we understand meaning as collaboration, we open a new vista of discussion. Every therapeutic school contributes to the discursive resources of the culture. Their distinct moves in the collaborative process of meaning-making offer possible departures from convention. In this sense, the plethora of therapeutic schools is not an embarrassment - somehow an indication of the pre-scientific status of the field. With the immense variations in cultural history from which clients emerge, we must cease to think in terms of a 'master conversation', useful to all. Rather, we have here a case in which multiple realities are to be valued.

To extend the argument, we must also recognize that therapeutic schools are themselves self-sustaining traditions. Typically they tend to reaffirm a particular vocabulary and honour certain moves in conversation over others. In this sense schools of therapy become culture-conserving. Yet, while the internal discourses of a school remain stable, the meaning-making process within the culture continues to evolve. New forms of coordination (and dis-coordination) are everywhere in motion. Today's profundity becomes tomorrow's platitude. Passions are cooled and values forgotten in the continuously unfolding illuminations generated by active and creative innovation. 
In this way we see that every school of therapy that sustains the dogmas of their founders and resists cultural changes is in danger of becoming irrelevant. Rather, the continuous evolution of therapeutic language and practice is necessary to sustain its relational efficacy. It is when therapeutic conversations are continuous with those of the culture that they will most effectively 'make sense'. It is when the client can coordinate the discourse of therapy with his or her life outside that therapy is most likely to succeed. As a result, new schools of therapy should not only be anticipated, but welcomed.

In conclusion, the present chapter has sketched the contours of what many see as major transformations in our understanding of truth, objectivity, rationality, morality, and progress. These transformations can conveniently be understood in terms of treatises on the social construction of the real and good. As these traditional assumptions are challenged, so can we move into new forms of understanding and practice. Here we have outlined a radically relational view of communication and understanding. Moving away from the traditional view of communication as intersubjective connection, we find meaning created within the spaces of coordination among persons. Thus, meaning is in continuous motion, over which no one has ultimate control. In this light, we took up a range of central issues in therapy. Issues of defining 'mental illness' and 'cure', client/therapist negotiation, the flow of meaning into and out of the therapeutic relationship, and the need for continuous transformation of the therapeutic process all demanded attention. In our view, these are scarcely the 'last words' on social construction and therapy. Rather, our hope is that they sustain what is now a global dialogue on discursive issues in therapy.

\section{References}

Edwards, D. and Potter, J. (1992). Discursive Psychology. Sage, London.

Fish, S. (1980). Is There a Text in this Class? The Authority of Interpretive Communities.

Harvard University Press, Cambridge, MA.

Gadamer, H. G. (1975). Truth and Method. The Seabury Press, New York.

Garfinkel, H. (1967). Studies in ethnomethodology. Prentice Hall, Englewood Cliffs, NJ.

Gergen, K. J. (2006). Therapeutic realities, collaboration, oppression and relational flow. Taos Institute Publications, Chagrin Falls, $\mathrm{OH}$.

Gergen, K. J. (2009a). An invitation to social construction (2nd ed.). Sage, London.

Gergen, K. J. (2009b). Relational being, Beyond self and community. Oxford University Press, New York.

Gergen, K. J. and Gergen, M. (2004). Social Construction, Entering the Dialogue. Taos Institute Publications, Cleveland, $\mathrm{OH}$.

Kuhn T. S. (1970). The Structure of Scientific Revolutions. Chicago University Press, Chicago, IL.

Latour, B. (1987). Science in action. Harvard University Press, Cambridge, MA.

Nightingale, D. J. and Cromby, J. (Eds.) (1999). Social constructionist psychology: A critical analysis of theory and practice. Open University Press, Buckingham.

Parker, I. (Ed.) (1998). Social constructionism, discourse, and realism. Sage, London.

Shotter, J. (1993). Cultural Politics of Everyday Life: Social Constructionism, Rhetoric, and

Knowing of the Third Kind. Open University Press, Milton Keynes. 
Spence, D. P. (1982). Narrative truth and historical truth, Meaning and interpretation in psychoanalysis. Norton, New York.

Wittgenstein, L. (1953). Philosophical Investigations (G. E. M. Anscombe, Trans.). The MacMillan Company, New York. 